\title{
Rechtsnatur der Gesetzgebungsakte von De-facto-Regimes: Ein Urteil des Obersten Gerichtshofes Uruguays
}

\author{
Von Franz-B. Marré
}

\section{Vorgeschichte}

Uruguay, einst als "Schweiz", in den siebziger Jahren als "Folterkammer" Lateinamerikas bezeichnet, verfügt seit fast vier Jahren wieder über demokratisch gewählte Staatsorgane. Doch die Spuren, die die mehr als elfjährige Militärherrschaft hinterlassen hat, sind tief und bedürfen einer Beseitigung nicht nur politischer Art.

Die juristische "Aufarbeitung" dieser Epoche begann kurz nach der Einsetzung der frei gewählten Gesetzgebungskörperschaften Anfang 1985: Der erste Gesetzgebungsakt eines frei gewählten Parlaments seit 1973 war ein Amnestiegesetz. Es trägt die Nummer 15.737; in ihm wurde eine Amnestie für alle politischen, gewöhnlichen und militärischen Straftaten, die seit dem 1.1.1963 begangen wurden, erklärt.1 Ausnahmen sollten nur für die grausame, unmenschliche oder erniedrigende Behandlung von Gefangenen sowie für das Festhalten verschwundener Personen gelten. Zweck dieses Gesetzes war es in erster Linie, Straftäter, die den Tupamaros (der in den 60er und 70er Jahren aktiven Terroristenorganisation) angehört hatten, von Strafe zu befreien. Lag dies ganz im Sinne der linken Opposition, so stieß das Vorhaben, auch die Militär- und Polizeikräfte von der Straf verfolgung auszunehmen, bei ihr auf erbitterten Widerstand. Gleichwohl wurde am 22.12.1986 das Gesetz Nr. 15.848 ("Ley de Caducidad") erlassen, wonach u.a. der staatliche Strafanspruch bezüglich Straftaten, die von Angehörigen der Streitkräfte und der Polizei bis zum 1.3.1985 begangen wurden, verfallen ist. Gegen die Art. 1-4 dieses Gesetzes ist von politischen Gruppierungen der Linken ein Volksbegehren in Gang gesetzt worden mit dem Ziel, sie per Referendum wieder aufzuheben, eine Möglichkeit, die Art. 79 Abs. 2 der Verfassung Uruguays von 1967 (UrVerf) vorsieht.2 Zur Zeit wird noch vom zuständigen Wahlgerichtshof ("Corte

1 Gesetz Nr. 15.737 vom 13.3.1985, "Diario Oficial" vom 22.3.1985.

2 Auch wurden Klagen gegen das Gesetz erhoben. In einem Anfang Mai 1988 gefällten Urteil, abgedruckt in "Revista Uruguaya de Derecho Constitucional y Politico", Bd IV, Nr. 24 (AprilMai 1988), bestätigte der Oberste Gerichtshof ("Suprema Corte de Justicia") mit drei zu zwei Richterstimmen die Verfassungsmäßigkeit der "Ley de Caducidad", weil die betreffenden Bestimmungen trotz ihrer grammatikalisch etwas mißglückten Fassung eine rechtlich nicht zu beantstandende Amnestie darstellten. 
Electoral") geprüft, ob die erforderliche Anzahl von Unterschriften (ein Viertel der Wahlberechtigten) vorhanden ist. 3

Am selben Tag wie das Amnestiegesetz, also am 13.3.1985, wurden durch das Gesetz Nr. 15.738, die "Ley de Convalidación"4, bis auf wenige, näher genannte Ausnahmen alle Legislativakte des "Consejo de Estado" (Staatsrat) bestätigt. Der Staatsrat war das von den Militärs statt des seinerzeit aufgelösten Parlaments geschaffene Gesetzgebungsorgan gewesen. Er hatte in der Zeit vom 19.12.1973 bis zum 14.2.1985 eine recht rege Tätigkeit entfaltet und fast 1600 "Gesetze" sowie mehrere "leyes fundamentales" und "leyes especiales" (Grund- und Sondergesetze) erlassen. Die von der Bestätigung ausgenommenen Akte betrafen nach der Begründung vor allem Gesetze, die entweder von offensichtlich repressivem Geist waren und den demokratisch-republikanischen Prinzipien der uruguayischen Verfassungsordnung widersprachen, sowie solche, durch die bestimmten Beamten, die das vergangene Regime repräsentiert hatten, außergewöhnliche Privilegien oder Vorteile gewährt worden waren. Die Verwaltung wurde angewiesen, Verwaltungsakte, die aufgrund dieser Normen erlassen worden waren, aufzuheben und im Einzelfall die Folgen der Aufhebung festzulegen.

Das Gesetz wurde im wesentlichen mit dem Bedürfnis nach Rechtssicherheit begründet. Zwar seien die betreffenden Akte des Staatsrates grundlegend nichtig ("radicalmente nulos"), da sie von einem Organ herrührten, das in der Verfassung nicht vorgesehen sei, und da sie unter Mißachtung des dort vorgeschriebenen Verfahrens zustandegekommen seien. Jedoch seien durch ihre jahrelange Anwendung eine Unzahl rechtserheblicher Beziehungen entstanden, so daß es unklug sei, diesen die rechtliche Grundlage zu entziehen. Allerdings bedürften diese Gesetzgebungsakte einer ausdrücklichen Bestätigung durch den verfassungsmäßigen Gesetzgeber, da sie andernfalls wirkungslos seien.

Das Parlament übernahm damit die Vorgehensweise seiner Vorgänger aus dem vergangenen Jahrhundert. In der zweiten Hälfte des 19. Jahrhunderts hatten mehrmals von den Streitkräften geführte "de-facto"-Regierungen die Geschicke des Landes bestimmt; die während dieser Zeit erlassenen "Gesetze" wurden nach Wiederherstellung der verfassungsmäßigen Ordnung vom regulären Gesetzgeber nachträglich sanktioniert. Dies war dagegen bei den beiden Staatsstreichen dieses Jahrhunderts (an denen die Streitkräfte nicht beteiligt gewesen waren) nicht der Fall, denn die neuen Verfassungen, die jeweils das Produkt dieser Putsche gewesen und durch Plebiszite angenommen worden waren, enthielten

3 Das Verfahren hat sich sehr in die Länge gezogen. Erst Ende 1988 wurde die Prüfung der Unterschriften durch die Corte Electoral mit der Feststellung abgeschlossen, da $\beta$ die erforderliche Zahl von 555.701 Unterschriften erreicht war. Das Referendum wird am 16. April 1989 stattfinden.

4 Etwa: "Anerkennungsgesetz", veröffentlicht im "Diario Oficial" vom 22.3.1985; im Anschluß an den Gesetzestext ist - für Uruguay einmalig - eine offizielle Begründung abgedruckt. 
in den Übergangsbestimmungen Klauseln über die "Ratifizierung" der vorangegangener Verfügungen in Form von Gesetzen und Verwaltungsakten.5

Die "Ley de Convalidacion" vom 13.3.1985 ist Anfang 1988 Gegenstand einer Entscheidung des Obersten Gerichtshofes Uruguays, der "Corte Suprema de Justicia" gewesen. Es handelt sich um das Urteil Nr. 1/88 vom 3.2.19886, das im folgenden vorgestellt werden soll.

\section{Das Urteil der Corte Suprema de Justicia vom 3.2.1988}

\section{Der Sachverhalt}

Dem Urteil zugrunde lag die verwaltungsgerichtliche Klage einer ehemaligen Universitätsbeamtin. Ihr war eine als "cargo de particular confianza" (Amt besonderen Vertrauens) bezeichnete Stellung übertragen worden. Es handelt sich dabei um einen besonderen Beamtenstatus, den das uruguayische Verfassungsrecht seit 1952 vorsieht. 7 Diese Beamten in besonderen Vertrauensstellungen sind nicht in die gewöhnliche Verwaltungslaufbahn einbezogen, sondem werden von den politischen Mandatsträgem in ihre Positionen - meist in der Spitze von Verwaltungskörperschaften - berufen und spätestens beim Wechsel des Politikers ausgetauscht.

Sie genießen also insbesondere nicht das den Beamtenstatus üblicherweise prägende Recht der Unabsetzbarkeit.8 Art. 93 ff. des noch unter der Militärherrschaft erlassenen "Sondergesetzes Nr. 7" vom 23.12.19839 hatte bestimmt, daß die entsprechenden Stellen aufzulösen seien. Jedoch wurde allen Inhabem solcher Ämter die Möglichkeit eingeräumt, für eine Umwandlung ihres Status in den eines Laufbahn- (d.h. Berufs-) Beamten zu optieren, ein vor allem angesichts der wirtschaftlichen Konsequenzen (Unkündbarkeit, Pensionsanspruch) nicht zu unterschätzender Vorteil.

Diese Umwandlungsbestimmungen wurden durch das Gesetz Nr. 15.738 für von Anfang an nichtig erklärt, was zur Folge hatte, daß die Antragstellerin ihres Amtes enthoben wurde. Mit einer Klage vor dem Verwaltungsgericht verfolgte sie das Ziel, daß diese Maßnahme wieder rückgängig gemacht werde. Nach Abweisung durch die erste Instanz war das Verfahren bei der zweiten und letzten Instanz, dem "Tribunal de lo Contencioso-Administrativo" (Verwaltungsgerichtshof) angelangt. Es wurde aber auf Antrag der Klägerin ausgesetzt, um zunächst durch den Obersten Gerichtshof, der u.a. die Funktion eines Verfassungsgerichts hat, die Frage klären zu lassen, ob dieses Gesetz Nr. 15.738 verfassungsge-

5 Vgl. hierzu: Alberto Ramón Real, Los Decretos-Leyes, Montevideo 1946, S. 253 ff.

6 Veröffentlicht in: Revista de la Facultad de Derecho y Sciencias sociales, Montevideo, año XXIX (Jan. - Juni 1988), S. 217 ff. Anm. v. José Anibal Cagnoni.

7 Vgl. heute Art. 60 Abs. 4 der uruguayischen Verfassung (UrVerf).

8 Vgl. Daniel Hugo Martins, Estatuto del funcionario, Montevideo 1965, S. 244 ff.

9 "Diario Oficial" vom 30.12.1983 
mäß sei, d.h. ob die fraglichen Vorschriften des "Sondergesetzes Nr. 7" tatsächlich "ex tunc" aufgehoben worden seien. 10

\section{Die Argumentation der Antragstellerin}

Die Antragstellerin meinte, es sei dem Parlament verwehrt, rückwirkend Gesetze aufzuheben. Hierzu sei ausschließlich die rechtsprechende Gewalt befugt, während der Gesetzgeber im Regelfall darauf beschränkt sei, Gesetze mit Wirkung "ex nunc" zu beseitigen. In keinem Fall könne eine Ausnahme dann gelten, wenn durch die Aufhebung wohlerworbene Rechte ("derechos adquiridos") betroffen würden. Um ein solches Recht handele es sich aber bei der von ihr eingenommenen beamtenrechtlichen Stellung. Aus Gründen der Rechtssicherheit und des Vertrauensschutzes sei es unzulässig, ihr diese zu entziehen. $11 \mathrm{Zu}$ Unrecht habe das uruguayische Parlament angenommen, die Gesetze des Staatsrates seien mit einem so schweren Fehler behaftet, daß sie nichtig seien. Zwar habe der Staatsrat die Kompetenz der Gesetzgebung nicht auf dem in der Verfassung vorgesehenen Wege erlangt. Er habe sich aber effektiv eingerichtet und seine Verfügungen seien als Gesetze behandelt und befolgt worden; es sei daher ein Gebot der Kontinuität des Staates und der Rechtssicherheit, ihnen weiterhin Gesetzescharakter zuzuerkennen. 12

Die beklagte Universität war der gegenteiligen Auffassung. Die funktionelle Unzuständigkeit des Staatsrates sei ein so gravierender Mangel, daß seine Akte nicht nur als fehlerhaft, sondem als nichtig anzusehen seien. Dieser Meinung schloß sich der Generalanwalt ("Fiscal de Corte", im weitesten Sinne vergleichbar mit dem Vertreter des öffentlichen Interesses im bundesdeutschen Verwaltungsprozeß) an, der eine Zurückweisung des Antrages empfahl.

10 Nach Art. 157 UrVerf hat der Oberste Gerichtshof die ausschließliche Befugnis zur Erklärung der Verfassungswidrigkeit von Gesetzen. Anders als in der Bundesrepublik, wo Voraussetzungen einer Verfassungsbeschwerde grundsätzlich die vorherige Erschöpfung des Rechtsweges ist $\S 90$ Abs. 2 BVerfGG - kann in Uruguay die Verfassungswidrigkeit einer Norm in jeder Lage eines gerichtlichen Verfahrens vor dem Obersten Gerichtshof geltend gemacht werden; in diesem Fall ist das Verfahren bis zur Entscheidung durch den Gerichtshof auszusetzen, Art. 258 Abs. 3 UrVerf.

11 Der Prozeßbevollmächtigte der Klägerin hat diese Position in zwei Aufsätzen in einer rechtswissenschaftlichen Zeitschrift vertreten und dort näher präzisiert: Daniel Hugo Martins, Validez y vigencia de los actos juridicos emanados del gobiemo de facto (1973-1985), in: Revista Uruguaya de Derecho Constitucionaly Politico (Rev. Ur. DCP) Bd. I, Nr. 6 (April-Mai 1985), S. 234 ff.; und ders., ¿Puede la ley declarar absolutamente nulos y anular actos legislativos vigentes?, in: Rev. Ur. DCP Bd. II, Nr. 7 (Juni-Juli 1985), S. 45 ff.

12 Martins, (Anm. 10), Rev. Ur. DCP Bd. I, Nr. 6, S. 234 (240). 


\section{Die Entscheidung des Gerichtshofes}

In einer einstimmig ergangenen Entscheidung hat der Oberste Gerichtshof den Antrag zurückgewiesen. Das Gesetz Nr. 15.738 sei verfassungskonform und damit wirksam.

a) Auf die Frage, ob das Parlament rückwirkend Gesetze aufheben könne, ging der Gerichtshof nicht ein. Nach seiner Ansicht setze dies voraus, da $B$ es sich bei den Akten, die durch das angefochtene Gesetz für nichtig erklärt wurden, rechtlich gesehen überhaupt um Gesetz gehandelt habe. Dies sei aber nicht der Fall gewesen: Bei den als "Gesetzen" bezeichneten Regelungen habe es sich lediglich um Tatsachen, nicht aber um rechtlich verbindliche Normen gehandelt. Das Gericht griff dabei zurück auf eine Unterscheidung des Zivilrechtlers Couture, wonach es drei Grade der Unregelmäßigkeit von Prozeßhandlungen gibt. die relative Nichtigkeit, d.h. Vemichtbarkeit, die absolute Nichtigkeit und die völlige Inexistenz bei besonders gravierenden Mängeln.13 Nach Auffassung des Gerichts hat hier ein der dritten Gruppe zuzurechnender Mangel vorgelegen: Durch das gesetzgeberische Tätigwerden eines Organs, das von einer "de-facto"-Regierung eingesetzt wurde, würden grundlegende Normen der Verfassung verletzt. Der Staatsrat habe sich indirekt die Ausübung der Souveränität angemaßt; diese stehe aber allein der Wählerschaft ("cuerpo electoral") und den von dieser bestimmten Repräsentativorganen zu (Art. 82 Ur.Verf). Das Regime, das unter Aussetzung der juristischen Normalität die Macht ausgeübt habe, habe diese Souveränität nicht legitimerweise beanspruchen können. Folge dieser Nichtexistenz sei, da $\beta$ die betreffenden Akte einerseits nicht nachträglich beseitigt werden könnten, es andererseits aber auch keiner ausdrücklichen Erklärung der Nichtigkeit bedürfe. 14 Insoweit habe das Gesetz lediglich deklaratorische Bedeutung.

b) Darüber hinausgehend äußert sich der Gerichtshof auch zu einer Frage, die für die unmittelbare Streitentscheidung nicht von Bedeutung ist: der Rechtsnatur der im Gesetz Nr. 15.738 vorgenommenen Bestätigung ("convalidación") aller nicht ausdrücklich ausgenommen Akte des Staatsrates. Da aufgrund der fundamentalen Fehlerhaftigkeit eine Bestätigung - etwa im Sinne der Genehmigung schwebend unwirksamer Rechtsgeschäfte - nicht möglich sei, habe es sich um die Neuverkündung mit rückwirkender Kraft gehandelt. Allein aus Gründen der Praktikabilität sei auf die Maßnahmen des Staatsrates verwiesen worden (Hier geht das Gericht weiter als das Parlament, zumindest drückt es sich deutlicher aus als dieses. Es hatte seinerzeit zwar von einer "grundlegenden Nichtigkeit" gesprochen, die

13 Eduardo J. Couture, Fundamentos del derecho procesal civil, 3. Auflage (posthum), Montevideo 1958, S. 376. Die Übertragbarkeit dieser Differenzierung auf das öffentliche Recht wird jedoch bestritten, vgl. Enrique Sayagués Laso, Tratado de Derecho Administrativo, Bd. 1, Montevideo 1953, S. 502, der letztlich allerdings anerkennt, da $B$ es - seltene - Akte gebe, die mit so schweren Fehlem behaftet seien, $\mathrm{da} B$ sie nicht als existent betrachtet werden könnten.

14 Dies ist nach Couture (Anm. 12), S. 378, dagegen bei "absolut nichtigen" Akten erforderlich, die bis zu dem Tag einer entsprechenden Feststellung eine Art "künstliches Leben" entfalteten. 
Begründung ließ jedoch die Möglichkeit einer Bestätigung eben jener Akte offen). Das Rückwirkungsverbot habe lediglich einfachgesetzlichen Charakter, da es nur im Zivilgesetzbuch, nicht aber in der Verfassung verankert sei. Daher sei es auch dem einfachen Gesetzgeber möglich, aus Gründen des allgemeinen Interesses, die hier auf der Hand lägen, davon abzuweichen.

c) Auch der Gesichtspunkt der Rechtssicherheit greife nicht ein. Aufgabe eines Rechtsstaates sei es gerade, den unter einem "de facto"-Regime entstandenen und daher illegitimen Akten die Anerkennung zu verweigem. Die Antragstellerin könne sich nicht darauf berufen, es habe sich um ein wohlerworbenes Recht gehandelt, auf dessen Bestand sie hätte vertrauen dürfen. Positionen, die außerhalb von Recht und Gesetz erworben worden seien, verdienten nicht die spätere Anerkennung, sobald der normale Zustand wieder hergestellt sei, sofem dem nicht Gründe des Allgemeinwohls entgegenstünden. Gerade dies sei aber hier der Fall gewesen: Der Gesetzgeber habe die Beseitigung von ungerechtfertigten und das Gleichheitsgebot verletzenden Privilegien und Ungleichbehandlungen bezweckt.

\section{Kritik}

So begrüßenswert die genannte Entscheidung im Ergebnis ist, wirft die Begründung doch einige Probleme auf, denen mit dem bloßen Hinweis auf die Illegitimität des vergangenen Regimes nicht ohne weiteres aus dem Weg gegangen werden kann. Das Gericht bemerkt zu Ende der Entscheidung, es halte es für 'unnütz und überflüssig, sich in Erwägungen über die von dem "de facto"-Regime, das seit 1973 das Land regierte, vertretenen Auffassungen und ihre Angemessenheit zu verlieren'. Gerade dies ist aber der Punkt, um den sich bei der Frage der Rechtsgeltung letztlich alles dreht.

Die "Suprema Corte" scheint generell Rechtshandlungen von Regierungen, die durch einen Umsturz an die Macht gekommen sind, sich aber nicht auf Dauer etablieren, für juristisch irrelevant zu halten. Damit widerspricht sie der überwiegenden Lehre, die im Anschluß an Constantineau 15 von der grundsätzlichen Wirksamkeit solcher Akte ausgeht, wenn auch meist die Möglichkeit einer Vernichtbarkeit angenommen wird.16 Bemerkenswert ist auch, $\mathrm{da} ß$ mit keinem Wort auf die Rechtsprechung im Nachbarland Argentinien eingegangen wird. Die dortige "Corte Suprema de Justicia de la Nación" hat sich im letzten halben Jahrhundert häufig mit der Frage der Rechtswirkungen solcher "de facto"-Gesetze zu befassen gehabt und die "de facto-Doktrin" Constantineaus dahin fortentwickelt, daß es für die Gül-

15 A Treatise on the De Facto Doctrine, Toronto, Rochester (N.Y.) 1910 (span. Ubersetzung Buenos Aires 1945).

16 Vgl. Sergio Deus, Validez y vigencia del Acto Institucional No.19, in: La Justicia Uruguaya, Bd. 90 (1985), Teil 2, S. 45 (47 ff.); Martins (Anm. 10), Rev. Ur. DCP Bd. I, Nr. 6, S. 234 (242 ff.), jeweils mit zahlreichen weiteren Nachweisen. 
tigkeit von Gesetzen gar nicht darauf ankomme, auf welche Weise das sie erlassende Organ eingesetzt wurde. 17

Ohne eine Differenzierung nach der Art des Regimes und vor allem nach dem Inhalt der fraglichen Entscheidungen dürfte die Ansicht des uruguayischen Obersten Gerichtshofes zu weitgehend sein und einige Folgeprobleme aufwerfen.

a) Der Zustand der Rechtssicherheit ist allerdings ein Kennzeichen des Rechtsstaates. Das Bedürfnis danach kann aber auch und gerade dann besonders groß sein, wenn bestimmten Handlungen ein Fehler anhaftet, sie sich also nicht im Rahmen der vom Staat gesetzten Legalität befinden. In solchen Fällen ist es wenig sinnvoll, wenn sich der Richter damit begnügt, allgemein auf die Irregularität des entscheidenden Organs hinzuweisen: Der Schutz individueller Rechtspositionen beginnt nicht erst in dem Augenblick, in dem sich eine rechtmäßig an die Macht gekommene Regierung etabliert. Vielmehr wird eine Wertung in zweierlei Hinsicht erforderlich sein: Bezüglich der Schwere des Mangels einerseits, hinsichtlich der Bedeutung des zu schützenden Interesses andererseits.

b) Bedenken begegnet ferner der Hinweis des Gerichts, das Parlament sei jederzeit befugt, Gesetzen rückwirkende Kraft zu verleihen.

Dies ist zwar ständige Rechtsprechung der "Suprema Corte de Justicia", sie wird aber in der innerstaatlichen Rechtslehre nicht ohne Grund kritisiert. Hingewiesen wird dabei einerseits auf den dem Art. 2 Abs. 1 GG entsprechenden Art. 10 Abs. 2 UrVerf ("Kein Bewohner der Republik wird verpflichtet zu tun, was nicht das Gesetz befiehlt, noch dessen beraubt, was es nicht verbietet."), daneben auf die Auffangklausel des Art. 72 UrVerf ("Die in der Verfassung vorgenommene Aufzählung von Rechten, Pflichten und Garantien schließt andere, die der menschlichen Persönlichkeit innewohnen oder die sich aus der republikanischen Regierungsform ableiten, nicht aus."). 18

In der Tat scheint es mit der Konzeption des Rechtsstaates schwerlich vereinbar zu sein, bei anderen belastenden Normen als Strafgesetzen - hinsichtlich letzterer ist das Verbot von "Ex post facto"-Gesetzen anerkannt 19 - die Zulässigkeit der Rückwirkung lediglich an Fragen des "Wohls der Allgemeinheit" zu knüpfen. Der Schutz nicht nur von Minderheiten, sondern auch des Vertrauens des Bürgers gebietet jedenfalls bei der "echten" Rückwirkung eine strengere Begrenzung, z.B. dadurch, daß nur zwingende Gründe des Gemeinwohls eine Rückwirkung rechtfertigen können. 20

17 Einen recht vollständigen Überblick über die Entwicklung der Rechtsprechung der "Corte Suprema" bis 1968 gibt Tilman Tönnies Evers, Die "Gesetzesdekrete" argentinischer Revolutionsregierungen, VRÜ 1 (1968), S. 333 (341 ff.).

18 Vgl. z.B. Alberto Ramón Real, Estado de derecho y humanismo personalista, Montevideo 1974, S. 51. Ähnlich wohl schon Justino Jiménez de Aréchaga, La Constitución Nacional, Montevideo 1946-49, Bd. II, S. $34 \mathrm{f}$.

19 Jiménez de Aréchaga, ebda. (Anm. 17).

20 Vgl. etwa BVerfGE 18, 429 (439). 
c) Angedeutet sei schließlich, daß die Entscheidung des Gerichtshofes auch in völkerrechtlicher Hinsicht Probleme aufwerfen könnte. Ginge man davon aus, daß die Ratifikationsakte des Staatsrates ebenso wie seine Gesetzgebungsakte nicht nur nichtig, sondem "nichtexistent" sind, so könnte die Regierung geneigt sein, völkerrechtliche Verträge aus der Zeit der "de facto"-Regierung als nicht geschlossen anzusehen. Die Mißachtung von Verfassungsrecht oder sonstiger innerstaatlicher Vorschriften kann aber nach der im Völkerrecht geltenden Evidenztheorie nur dann zur Anfechtbarkeit eines Vertrages führen, wenn der Vertragspartner den Mangel erkannte oder nach Treu und Glauben hätte erkennen müssen.21 Diese Voraussetzung kann sich freilich nur auf die jeweils effektiv gültige innerstaatliche Rechtsordnung beziehen, so daß eine Anfechtbarkeit ausschiede.

\section{Schlußbemerkung}

Die Auswirkungen der Zeit des Militärregimes werden die uruguayische Justiz noch auf Jahre hinaus beschäftigen, zumal die Entscheidungen der "Suprema Corte" auch in Fragen der Verfassungsmäßigkeit von Gesetzen lediglich den konkreten Fall betreffen (Art. 259 UrVerf). Entgegen der oben wiedergegebenen Argumentation der Antragstellerin ist eine gerichtliche Aufhebung von Gesetzen also gar nicht möglich. Vermutlich wird die Entscheidung aber den Charakter eines "leading case" bekommen, denn ihre Bedeutung reicht weit über den entschiedenen Sachverhalt hinaus. Eine Beseitigung der Probleme des Landes läßt sich dadurch aber kaum bewirken.

Letztlich ist die Situation Uruguays weitgehend identisch mit der des Jahres 1973, sieht man einmal von der Tatsache ab, daß die "Nationale Befreiungsbewegung - Tupamaros" inzwischen als legale Organisation existiert und keine bewaffneten Aktivitäten mehr ausübt. Die Ursachen der damaligen Krise sind nicht beseitigt worden. Um diese zu bewältigen sind aber - zunächst - die Politiker gefragt, nicht die Juristen.

21 Vgl. Art. 27 und 46 der Wiener Vertragsrechtskonvention vom 23.5.1969 (BGBL. 1987 II, S. 757), in Uruguay seit dem 4.4.1982 in Kraft, sowie aus dem Schriftum Alfred Verdross/ Bruno Simma, Universelles Völkerrecht, 3. Auflage, Berlin 1984, S. 443 ff. (\$§ 689 ff.). 


\title{
ABSTRACTS
}

\author{
The Latin American Tradition of Legitimising Coup d'état Governments: the \\ Example of Argentina
}

By Wolfgang Spoerr

Analysing the development of this de-facto doctrine in the case law of the Argentine Supreme Court since 1930 and of the arguments of Argentine legal theorists, it is submitted that no logically consistent constitutional foundation of the doctrine has been found so far. The doctrine reflects the desire of lawyers (both judges and academics) to keep the military governments under some legal control and to preserve the integrity of the legal order so far as possible and, furthermore, the practical need to give at least some recognition to the acts of de-facto governments even after the retum to constitutional rule. The desire to keep the military governments within some legal boundaries has proved to be an illusionary goal, given the fact that the prerogatives recognised by the courts have been extended in each of the five periods of military rule since 1940 . After the most recent retum to democracy, however, there have again been tendencies to restrict the validity of these govemments' acts. To accuse the judiciary in general of undermining the rule of law would, however, not be justified by Argentine history. The legal system is not in a position to prevent the deterioration of a constitutional order which is brought about by extra-legal developments, but a functioning and accepted judiciary is important as one of the key conditions of democratic stability.

Legal Nature of Legislative Acts by de-facto Régimes: A Judgment by the Uruguayan Supreme Court

\section{By Franz-B. Marré}

Uruguay is again, since 1985, governed by democratically elected authorities after an eleven-year period of military rule. In order to prevent a legislative vacuum the new Parliament confirmed most of the more than 1.600 "laws" enacted by the de-facto régime. Nevertheless, this measure expressly excluded laws of repressive character and those contradicting the essential principles of the Uruguayan constitution. In early 1988 the Supreme Court dealt with the derogation of these acts. Its judgment approved the parliamentary approach arguing that the "laws" issued by the military govemment were mere 
factual acts, incurably null and legally void. From a formal point of view, the confirmation would have to be seen as a completely new enactment with retroactive effect. According to the Supreme Court the exclusion of certain statutes was not to be objected to, particularly not for a violation of the principle of legal certainty nor for the protection of vested rights. The author in substance welcomes the decision. His objections point towards the somewhat superficial treatment of problems concerning the rule of law, and the non-consideration of implications for public international law.

\section{The Influence of the New Law of the Sea on Cuba's Maritime Zones and Fishing} Policy

\section{By Beate Ratter}

After the signing of the Convention on the Law of the Sea in Jamaica in 1982 it will be interesting to ask about the effects of this new international instrument on national policies. There is, in fact, considerable influence not only on the legislation of each country but also on their economic development. The Cuban policy, of extending maritime zones and reorganizing the fishing industry, will be an example of the dimension of this impact.

Nearly all the regulations of the new convention are part of the Cuban legislation, i.e. the Straight Baseline, $12 \mathrm{~nm}$ Territorial Sea, and the $200 \mathrm{~nm}$ Exclusive Economic Zone (EEZ). In connection with the extension of the Cuban Territorial Sea and the reclamation of an Exclusive Economic Zone the Cuban government had to negotiate with its neighbours the extent of these limits in the sea. Treaties were signed with Mexico (1976), with Haiti (1977), and with the United States (1977). The frontiers with Honduras, the Cayman Islands, Jamaica and the Bahamas are not yet under negotiation and therefore the delimitation in respect of these countries needs further clarification.

Besides these changes, the new provisions of the Law of the Sea Convention had an essential influence on the fishing industry in Cuba. Formerly the Cuban fleet war used to exploit international fishing grounds in the Southeast Pacific, the North and South Atlantic which now are no longer international waters because of the proclamation of a $200 \mathrm{~nm}$ EEZ in different parts of the world. The affected Cuban fishing industry decided to improve the exploitation of their own fishing grounds around Cuba and finally they were successful in concentrating their activities in their own EEZ. 